\title{
La videoeducazione per la formazione degli insegnanti: sviluppi e prospettive nel Web 2.0
}

\section{Silvia Micheletta}

Università degli Studi di Firenze, Dipartimento di Scienze della Formazione e Psicologia doi: 10.7358/ecps-2014-010-mich silvia.micheletta@unifi.it

\section{TEACHER TRAINING COURSE ENHANCED BY VIDEO: DEVELOPMENTS AND PERSPECTIVES IN THE WEB 2.0}

\section{Abstract}

In the broadest sense, video-based education has a long history that dates back to the last century. The spread of television and later of the multimedia have profoundly marked the increased use of video in education and teaching. In recent years, digitization and broadband technology as well as the increasingly widespread availability of mobile technology, which is economical and easy-to-use, have transformed the web into a huge sphere for video production and sharing. The phenomenon now takes on a new aspect for education and looks set to significantly change teacher training methods. In this paper we describe the new prospects of video-based education brought by recent web developments, with particular reference to Web 2.0 with YouTube and other video-sharing platforms. We will present two training methods enhanced by videos, video-modeling and video-coaching, and we will analyze their potential problems with reference to teacher training.

Keywords: Digital video, Teacher training, Video-coaching, Video-modeling, Web 2.0. 


\section{INTRODUZIONE}

Con il termine videoeducazione ${ }^{1}$ si fa riferimento ad un complesso di interventi educativi che trae vantaggio dalla visione di video. In un'accezione così lata, la videoeducazione ha una lunga storia che risale alle prime proiezioni cinematografiche dell'inizio del Novecento, portata avanti grazie alla diffusione della televisione e, successivamente, dallo sviluppo della multimedialità. Recentemente la digitalizzazione, la banda larga e la miniaturizzazione dei dispositivi (sia dei supporti di memoria che delle tecnologie vere e proprie) hanno determinato un fortissimo incremento dei video, non solo in termini di fruizione a fini educativi, ma anche in termini di produzione. L'avvento del Web 2.0 (O'Reilly, 2005), infine, ha prodotto una vera e propria esplosione dell'uso dei video, creando nuovi formati impiegabili a scopo educativo e stimolando nuove istanze formative.

Sin dalla loro nascita i video offrono una molteplicità di vantaggi e altrettante criticità a livello educativo e didattico. Il video consente, ad esempio, di far vedere analiticamente azioni complesse (un macchinario da lavoro, un'esecuzione di un acrobata), ma anche ciò che per varie ragioni - lontananza spazio/temporale - non si può osservare direttamente (un fenomeno atmosferico, il comportamento di un animale), oltre che di «conservare» il fenomeno osservato per poterlo riesaminare in luoghi e momenti differenti. Il video, essendo costituito da immagini, consente spesso di superare le difficoltà e le ambiguità che caratterizzano il linguaggio verbale, sia scritto che orale, facilitando l'osservazione diretta e pratica (non è un caso che talvolta venga utilizzato con soggetti con disabilità, ad esempio casi di autismo). Allo stesso tempo non si devono sottovalutare le criticità che avvolgono la rappresentazione visiva, soprattutto quella dinamica mediata dai video. A questo proposito, molti autori hanno avanzato una serrata critica intorno alle ingenuità che si accompagnano all'uso educativo del video, fonte di possibile distrazione, distorsioni o sovraccarico cognitivo (Sweller, 1988; Mayer, 2001).

I video possono essere utilizzati dagli studenti come oggetto direttamente fruibile (ad esempio un video documentario) o come oggetto prodotto ( $\mathrm{ad}$ esempio un telegiornale realizzato direttamente dagli studenti). Si trascurano solitamente altri impieghi del video educativo, come quelli che riguardano la formazione degli insegnanti, vale a dire le ricerche e le esperienze che possono rendere tale formazione più efficace ed efficiente, tema che sarà oggetto del presente contributo.

1 Il termine italiano «videoeducazione» può essere tradotto in inglese con espressioni tipo video-based training o video-enhanced training, sebbene sia pur valida anche l'espressione video education. 
Come noto la formazione degli insegnanti è oggetto di grandissimo interesse nelle politiche internazionali (European Commission, 2002 e 2013; OECD, 2005 e 2009; UNESCO, 2005; ILO, 2010; Dordit, 2011) ${ }^{2}$. All'interno di questo ambito acquista sempre più particolare importanza, anche alla luce delle recenti risultanze dell'Evidence Based Education (EBE) (Hargreaves, 1997; Calvani, 2007 e 2012; Ranieri, 2007; Hattie, 2009 e 2012), la qualità dell'interazione didattica, un aspetto composito e articolato. In un campo complesso come quello educativo il video apre nuove prospettive per la formazione insegnanti che oramai non accetta più di appellarsi solo alla comunicazione verbale, ma richiede un adeguato supporto da parte della dimensione visiva, attraverso l'osservazione diretta.

Generalmente si distinguono tre metodologie di formazione insegnanti supportate dal video: il video-viewing, solitamente usato per acquisire ed esercitare metodi di osservazione e analisi; il video-modeling, generalmente prodotto da altri insegnanti, più o meno esperti, volto a fornire una dimostrazione pratica di competenze e comportamenti da acquisire; il video-coaching, creato dagli insegnanti stessi e fonte di discussione e confronto (tra pari e/o con mentore) (Masats \& Dooly, 2011).

In questo contributo ci soffermeremo ad analizzare potenzialità e criticità del video-modeling e del video-coaching per la formazione degli insegnanti, alla luce dei recenti sviluppi conseguenza del grande cambiamento generato dall'avvento del Web 2.0.

\section{I VIDEO SU INTERNET}

La maggior parte del traffico internet di tutto il mondo - quasi la metà negli Stati Uniti, e una percentuale molto più alta a livello globale - è composto da file audiovisivi (Kaufman \& Mohan, 2009).

Secondo una recente indagine condotta da Accenture (2012), la combinazione di un pubblico sempre più online, l'adozione di nuove tecnologie più versatili e accessibili e l'elevata disponibilità di contenuti spiega l'alto tasso di consumo di video online.

I video su internet sono a dir poco una rivoluzione nel settore. Molti operatori di tutto il mondo stanno facendo investimenti significativi per posizionarsi con

2 Recentemente negli Stati Uniti il progetto MET (Measures of Effective Teaching), finanziato dalla Fondazione Bill \& Melinda Gates, ha studiato la misura dell'efficacia dell'insegnamento e la valutazione della qualità degli insegnanti. Il progetto, tra l'altro, prevedeva di videoregistrare gli insegnanti affinché diversi osservatori potessero visionare le lezioni in tempi e luoghi diversi. 
successo in questo nuovo spazio. [...] Il video su internet è oramai diventato una parte consolidata di abitudini per la maggior parte dei consumatori, in molti paesi e in tutti i gruppi di età. [...] Mentre la piattaforma del video su internet matura, stanno sorgendo alcune domande, opportunità e sfide chiave per i provider [...], produttori di contenuti, produttori di tecnologia di consumo e nuovi business su internet che stanno cercando di capitalizzare questo spostamento da media di nicchia a media d'uso comune. ${ }^{3}$ (trad. libera)

L'indagine in questione (ibid.), condotta a livello internazionale ${ }^{4}$, rivela che il 92\% della popolazione guarda video online, dimostrando un forte aumento rispetto allo stesso dato dell'anno precedente $(77 \%)^{5}$.

I recenti sviluppi tecnologici, la semplicità d'uso e la diffusione di nuovi dispositivi mobili (quali personal computer, smartphone, tablet) ${ }^{6}$, così come le fotocamere e le videocamere digitali, consentono a un numero sempre più vasto e diversificato di persone, anche a chi non è del mestiere, la possibilità di produrre e condividere video (Bonaiuti, 2010).

Nel 1995 Negroponte già si prefigurava parte di questo scenario.

In questo contesto, ogni persona può essere considerata una stazione TV non autorizzata. Nel 1993 negli Stati Uniti sono stati venduti tre milioni e mezzo di videocamere. Ogni filmato casalingo non sarà, grazie al Cielo, un capolavoro. Però, d'ora in poi, il valore di un filmato non dovrà essere giudicato soltanto in base al livello di professionalità con cui è stato prodotto. [...] Tra pochi anni imparerete a fare il couscous da una cuoca famosa o da una casalinga marocchina. Scoprirete i vini con un famoso sommelier o con un vignaiolo della Borgogna. (ivi, p. 184)

3 «Video over broadband is nothing short of an industry revolution. Many operators around the globe are making significant investments to position themselves for success in this new space. [...] Video over the Internet has become an established part of viewing habits for most consumers, in most countries and across all age groups. [...] However, as the platform matures it is starting to generate interesting questions, opportunities and challenges for providers (both broadcasters and cable/telco operators), content owners, consumer technology manufacturers and new Internet businesses looking to capitalize on the move from niche to mainstream» (Accenture, 2012, p. 3).

4 L'indagine si è svolta su un campione di 7.503 utenti della rete. I paesi coinvolti sono: Argentina, Brasile, Francia, Germania, Italia, Spagna, Regno Unito e Stati Uniti.

5 Dai dati di Audiweb Objects Video: nel mese di aprile 2014 in Italia 9 milioni di utenti hanno visualizzato almeno un contenuto video online, per un totale 136 milioni di stream views, spendendo in media 58 minuti per persona (Audiweb, 2014b). Questi dati rivelano un aumento rispetto alle stesse rilevazioni effettuate solo due mesi prima (febbraio 2014), dove gli 8,6 milioni di utenti connessi che hanno visualizzato almeno un video online per un totale di 115 milioni di stream views hanno speso in media circa 41 minuti per persona in quest'attività (Audiweb, 2014a).

6 Nel 2012 ben il 46\% della popolazione statunitense (sopra i 18 anni) ha acquistato uno smartphone (Zickhur \& Smith, 2012). 
La produzione dei video è favorita, oltre che dalle sempre più prestanti potenzialità tecnologiche dei dispositivi mobili ${ }^{7}$, anche dalle nuove opportunità d'uso del video digitale (essenzialmente cattura, editing, riproduzione), dalla diffusione dei social network e l'app (da application, ossia applicazione) a "forte impatto visivo» (Facebook ${ }^{8}$, Pinterest ${ }^{9}$, Instagram ${ }^{10}$, Snapchat ${ }^{11}$, FrontBack ${ }^{12}$, Vine ${ }^{13}$ ), e dall'esplosione di spazi di rete per la condivisione, come YouTube, Vimeo ${ }^{14}$.

\section{Il VIDEO-MODELING NELL'ERA DEL Web 2.0: YouTube}

YouTube è la più diffusa piattaforma di video-sharing. Sin dalla sua nascita (febbraio 2005) ottiene subito un notevole successo tanto che nel 2006 viene acquisita da Google Inc.

È un'imponente risorsa che mette a disposizione una vastissima gamma di video, catalogati e indicizzati e che offre la possibilità di condividere sia in ambiente pubblico, che privato, i video prodotti e ospitati gratuitamente. È il sito web più visitato al mondo dopo Google e Facebook (è diffuso in 61 paesi e disponibile in 61 lingue), con 157,585 milioni di utenti e con oltre 12 milioni di video visualizzati (Jarboe, 2014), presenta il maggior tasso di crescita di visite: ogni mese sono visualizzati oltre 6 miliardi di ore di video $(2 \mathrm{mi}-$

7 Tra le azioni più frequenti fatte con tablet o smartphone, oltre all'inviare e ricevere sms ed e-mail, vi sono anche il registrare un video (dato raddoppiato rispetto al 2007), guardare un video (più che raddoppiato rispetto al 2007), inviare il video a un amico, postare o caricare un video online (Zickuhr \& Smith, 2012).

8 Facebook, nato nel 2004, è uno dei più noti e diffusi social network. Principalmente consente la pubblicazione e condivisione di commenti e pensieri, documenti, note, foto e video all'interno della propria bacheca personale o su gruppi più o meno aperti.

9 Pinterest è un social network fondato nel 2010, incentrato sulla condivisione di immagini e video.

10 Instagram è un'applicazione gratuita che consente di condividere su altri social network (tra cui Facebook) foto con svariati filtri.

11 Snapchat è un'applicazione di Instagram realizzata per smartphone che consente di inviare clip video della durata di 15 secondi.

12 FrontBack è un'applicazione pensata per gli smartphone che hanno la fotocamera anteriore e quella posteriore, in cui vengono condivise immagini della duplice prospettiva.

13 Vine è un'applicazione gratuita di Twitter che permette di creare video di breve durata (6 secondi) e di condividerli sui social network.

14 Vimeo, fondato nel 2004 da un gruppo di cineasti con lo scopo di condividere il loro lavoro creativo e momenti personali dalla loro vita, è il principale sito di video-sharing che concorre con YouTube. Come YouTube, Vimeo consente la possibilità di condividere, pubblicare e commentare i video su altri siti. Al contrario di YouTube, Vimeo pubblica solo opere video create dagli utenti. 
liardi di ore in più rispetto allo scorso anno) e ogni minuto vengono caricate 100 ore di video (poco più di 4 giorni) (YouTube Statistics, 2014). Inoltre, solo nel corso del 2011, il sito ha registrato più di un trilione (un milione alla terza!) di visualizzazioni, pari a circa 140 visualizzazioni per ogni persona del mondo (ibid.) ${ }^{15}$. Negli ultimi anni la visione dei video online è in forte aumento anche sui dispositivi mobili: l'app di YouTube è disponibile su centinaia di milioni di dispositivi mobili che coprono quasi il 40\% (aumentato del 6\% rispetto allo stesso dato del 2011) del tempo di visualizzazione totale della piattaforma (ibid.).

La caratteristica che più distingue YouTube da altre piattaforme di video-sharing è la sua semplicità di uso, visione, caricamento e incorporamento video ${ }^{16}$. Per ricercare, vedere e incorporare i video di YouTube in un sito, un documento o altro non è necessario essere iscritti; per caricare, condividere video e iscriversi ai vari canali basta avere un account di Google e/o un canale YouTube. L'iscrizione ai canali - vi è una vastissima gamma di canali su YouTube che spazia dallo sport alla letteratura, dai giochi all'informazione politica, dalla musica alla cucina - consente di rimanere aggiornati sui nuovi video pubblicati su quei particolari canali e di seguire i temi, per i quali abbiamo espresso preferenza, anche su canali affini. Costituendo un "canale» personale, invece, si ha la possibilità di caricare video su YouTube e di condividerli a vari livelli: aperta a tutti o aperta a un gruppo definito di persone (anche una persona sola) e nascosta dunque a tutti gli altri.

La maggior parte dei contenuti è costituita da video creati dai singoli utenti (i video che riproducono materiale di terzi senza autorizzazione vengono eliminati), ma sono diverse e sempre più numerose le organizzazioni e gli enti che consentono la visualizzazione dei loro video (ad esempio trasmittenti televisive, università, istituzioni, organizzazioni). Per fare qualche esempio: Rai, La7, BBC, MIT, CERN, European Commission, MIUR, Università degli Studi di Firenze, hanno tutti un proprio canale su YouTube.

Date le numerosissime risorse navigabili in YouTube e la volatilità del web, negli anni si sono costituiti vari servizi online, programmi ed estensioni per il browser, che consentono di scaricare in maniera gratuita i video di YouTube in differenti formati (ne sono un esempio SaveMedia e KeepVid).

15 Per dare un'idea del potere dell'enorme community di YouTube è curioso sapere che questa contribuisce, attraverso il numero delle visualizzazioni (una visualizzazione per ogni utente che ha guardato il video in questione), a far diventare «famosi» gli utenti più cliccati. Il fenomeno delle «celebrità di YouTube» è in piena ascesa grazie anche alla possibilità di diventare partner del sito, traendo guadagno dalle pubblicità e dalle visualizzazioni degli utenti. Tutto ciò è indicativo di quanto il fenomeno dei video digitali sia in costante ascesa.

16 Incorporare video significa inserire il link e visualizzare il video stesso all'interno di blog, social network e altri siti web. 
$\mathrm{Al}$ proprio interno YouTube offre diverse sezioni specifiche dedicate interamente all'educazione. Queste sono: YouTube EDU, YouTube Teachers, e la nuova YouTube for Schools.

YouTube EDU, come YouTube generico, non richiede alcuna iscrizione: è sufficiente accedere al sito per poter visualizzare i video caricati e incorporarli in documenti, blog o siti. Come YouTube, anche YouTube EDU offre la possibilità di creare un account, consentendo l'iscrizione ai canali e offrendo la possibilità di commentare i video e di avere suggerimenti su altri video interessanti.

YouTube EDU contiene oltre 700.000 video didattici supportati da più di 800 canali di organizzazioni famose come l'Università di Stanford, Public Broadcasting Service Learning Media (PBS), TED, e da partner emergenti con milioni di visualizzazioni, come Khan Academy, Steve Spangler Science e Numberphile. YouTube EDU è articolato in tre sezioni specifiche per ogni fase di apprendimento della vita: "Primary e Secondary Education» ${ }^{17}$ (istruzione primaria e secondaria), "Higher Education» (università) e "Lifelong Learning» (apprendimento permanente). Nella sezione "Primary e Secondary School Education" sono raggruppati i video che fanno riferimento alla fascia d'età compresa tra i 6 e i 18 anni, suddivisi per aree tematiche: lingua, matematica, scienze, storia, geografia e scienze sociali. Nella sezione «Higher Education» sono raccolti i video che afferiscono alla formazione universitaria di diverse aree tematiche tra le quali: matematica, scienze sociali, storia, studi umanistici, arte, ingegneria, scienze, diritto, affari, istruzione, medicina, lingue (ognuna con una serie di ulteriori canali al proprio interno). Nella sezione «Lifelong Learning» vi sono tutti i video che possono rientrare nella categoria della formazione permanente ossia, dell'educazione degli adulti.

Per ciascuna delle aree tematiche sopra menzionate, YouTube EDU fornisce delle playlist di video su particolari argomenti o suggerisce dei video già settati in un corso online. Ne sono un esempio un corso sulla Costituzione americana costituito da 42 video all'interno dell'area tematica della storia nella sezione dell'istruzione primaria e secondaria ${ }^{18}$, un corso sui differenziali matematici nell'area tematica della matematica per l'istruzione primaria e secondaria (costituito da 9 video) ${ }^{19}$, un corso sulla chimica costituito da

17 Secondo il sistema scolastico americano con primary e secondary education si fa riferimento alla primary school (scuola primaria), alla middle school (scuola secondaria di primo grado) e alla high school (scuola secondaria di secondo grado).

18 https://www.youtube.com/playlist?list=PLi3U-nPPrbS5d-juhFwo3hTBso0gq2sUZ (consulted 08/2014).

19 https://www.youtube.com/playlist?list=PLOa7j0qx0jgPTORxxrhPbX2XX6YWm98qy (consulted 08/2014). 
71 video all'interno dell'istruzione universitaria ${ }^{20}$ e un corso sulle pratiche di riflessione costituito da 10 video realizzati da MIT all'interno dell' istruzione universitaria $^{21}$.

YouTube Teachers, invece, è stato creato appositamente per aiutare gli insegnanti ad utilizzare didatticamente i video di YouTube. A questo scopo propone oltre 400 playlist video su una vasta gamma di argomenti: ad esempio le operazioni e la geometria nella scuola dell'infanzia, lo studio dell'apparato digerente umano, fino ad arrivare alla spiegazione delle equazioni complesse. Gli insegnanti, dunque, possono utilizzare le playlist create da altri membri della community o crearne delle nuove (solo se registrati al sito), o, più semplicemente, trarne spunto.

YouTube for Schools, infine, è una sezione a parte di YouTube pensata esclusivamente per le scuole. Una scuola, registrandosi su YouTube for School, ha possibilità di accedere e personalizzare le centinaia di migliaia di video didattici fruibili da YouTube EDU, creando un sito di navigazione sicura per i propri studenti (i commenti e i video correlati sono disattivati).

Oltre YouTube vi sono altri siti di notevole rilievo che indicizzano e catalogano numerosi video da utilizzare nella didattica o per la formazione e l'educazione in generale, come $P B S, T E D$, o meglio la sua sezione $T E D-E d$, e Teaching Channel.

PBS, Public Broadcasting Service, è un'azienda no-profit statunitense costituita da un consorzio di numerose stazioni televisive pubbliche nazionali, che ha lo scopo di divulgare contenuti che educhino e informino. Per questo motivo offre una programmazione molto ricca, sia per adulti che per bambini, di documentari, telegiornali non commercializzati e programmi di musi$\mathrm{ca}$, teatro, danza e arte. Al proprio interno, $P B S$, oltre ad avere una sezione specifica rivolta ai bambini («PBS Kids»), offre due importanti sezioni: una dedicata ai genitori(«PBS Parents») e una rivolta agli insegnanti («PBS LearningMedia»).

«PBS LearningMedia» offre circa 30.000 risorse digitali (video, file audio, documenti, risorse interattive e progetti di lezioni), tutti allineati con gli standard americani, da poter utilizzare nei differenti gradi e ordini di scuola: lo scopo è migliorare l'efficacia dell'insegnante e le acquisizioni degli allievi. Il sito consente la ricerca all'interno del catalogo per obiettivi, livello scolastico e area disciplinare. Le risorse sono accessibili a chiunque, ma solo agli utenti iscritti al sito è consentito salvare, condividere e scaricare, ove permesso, le risorse utilizzate.

20 https://www.youtube.com/playlist?list=PLllVwaZQkS2op2kDuFifhStNsS49LAxkZ (consulted 08/2014).

21 https://www.youtube.com/playlist?list=PL773D0AF1A86E4E58 (consulted 08/2014). 
PBS offre anche un'intera sezione ricca di video («PBS Video») all'interno della quale si possono trovare i video dei programmi delle stazioni televisive consorziate, la loro programmazione e una watchlist, creabile solo dagli utenti iscritti, in cui è possibile salvare una lista di video di cui tenere traccia.

$T E D$, un'organizzazione no-profit nata nel 1984 dall'unione di tre mondi, Technology, Entertainment e Design, si ispira al concetto di «Ideas Worth Spreading» (letteralmente: le idee che vale la pena diffondere). TED-Ed, la sezione rivolta a insegnanti e studenti, offre video educativi, molti dei quali sono oggetto di collaborazioni tra educatori e animatori nominati attraverso la piattaforma stessa. Rispetto a YouTube, TED-Ed consente a tutti gli utenti iscritti di poter creare direttamente sulla piattaforma una lezione attorno ad un video selezionato su TED o YouTube, con la possibilità di tenerla privata o renderla pubblica a tutta la community TED-Ed. Una TED-Ed Lesson è costituita dal video (visibile sotto la sezione "Wacht»), da una prova di verifica (da completare direttamente nella sezione «Think»), una serie di spunti di approfondimento relativamente al tema trattato (disponibili nella sezione "Dig Deeper») e da un'area discussione (accessibile dalla sezione "Discuss»). Alla presentazione della TED-Ed Lesson vi sono anche altri video correlati al tema. Oltre a tutto ciò, TED-Ed offre una sezione («Series») in cui vi sono playlist di video già create su particolari argomenti.

Teaching Channel, un'organizzazione no-profit statunitense lanciata nel 2011, offre una serie di video atte a mostrare le principali pratiche di insegnamento efficaci. I video offerti, prodotti da un unico team di professionisti, affrontano una vasta gamma di argomenti per studenti di diversi ordini e gradi scolastici (inclusa l'educazione speciale), in linea con il Common Core statunitense. Rispetto a YouTube, piattaforma di condivisione video che potremmo definire generalista, Teaching Channel è uno strumento specifico, pensato apposta per gli insegnanti, e volto a offrire loro diverse funzionalità, tra le quali: la possibilità di filtrare i video per ordine e grado scolastico, disciplina e argomento, un'area di discussione, alcune domande guida per l'analisi dei video, la possibilità di annotare commenti personali e di confrontarsi con altri membri della community (si veda il par. successivo).

A questi si possono aggiungere archivi video di centri di ricerca attivi nell'Evidence Based Education. Questi si impegnano a fornire dimostrazioni video di modelli di pratiche didattiche efficaci. Ne sono un esempio Edutopia, un progetto della George Lucas Educational Foundation, e l'Education Endowment Foundation che cura il Teaching and Learning Toolkit in cui vi è un archivio di video e studio di casi.

Di notevole interesse è anche il sito TIMSS che mette a disposizione una serie di video lezioni raccolte come parte degli studi sul campo TIMSS. Questi video sono proposti con molteplici scopi: forniscono una base concre- 
ta per l'interpretazione dei risultati quantitativi dello studio TIMSS, dimostrazioni concrete dei risultati riportati nei report ${ }^{22}$.

Diversi progetti sono stati avviati con l'obiettivo di valorizzare l'uso dei video disponibili sul web al fine di sostenere le attività di apprendimento (Gutiérrez Martín, 2011).

Prendendo come riferimento lo scenario europeo, sono state avviate diverse iniziative, come ad esempio Viducate, OnAir e EduTubePlus. Il primo è un progetto orientato a insegnanti e studenti delle scuole secondarie, il cui scopo principale è quello di esplorare l'uso di video per sviluppare la cittadinanza attiva in contesti interculturali a tutti i livelli di istruzione formale e informale. Il progetto OnAir è stato co-finanziato nel quadro del programma di apprendimento permanente (Programma Comenius Sub) al fine di promuovere un'efficace applicazione dei media nell'educazione (in particolare l'alfabetizzazione, leggere e scrivere). Infine, il progetto EduTubePlus è stato co-finanziato nell'ambito del programma europeo eContentplus con l'obiettivo di sviluppare un servizio ibrido multilingua video-based per le scuole, attraverso la creazione di strumenti che consentano ai docenti di arricchire l'archivio con contenuti generati dagli utenti e di recuperarli per mezzo di un motore di ricerca basato su un'antologia multilingue di termini correlati al curriculum.

Università in tutto il mondo (come la University of Berkeley e la Yale University) hanno creato un proprio canale di YouTube e utilizzano questo tipo di supporto per fornire informazioni e contenuti di apprendimento per i loro utenti. Ad esempio, il progetto Coursera costituisce una società imprenditoriale che collabora con alcune delle migliori università del mondo (come la Princeton University e la University of Stanford), al fine di offrire corsi online gratis che coprono una vasta gamma di argomenti. Recentemente, il progetto $E d X$ costituisce un'impresa no-profit, fondata dalla Harvard University e il $M I T$, con l'obiettivo di offrire corsi online su varie discipline e indagare come gli studenti imparino e come la tecnologia possa trasformare il loro apprendimento.

22 «Dissemination of these public use lessons serves multiple purposes. The videos provide a concrete basis for interpreting the quantitative findings of the TIMSS 1999 Video Study. They provide illustrations of key findings that communicate more clearly than written reports or oral presentations alone» (http://www.timssvideo.com/about-site - consulted 05/2014). 


\section{IL VIDEO-COACHING: STRUMENTO DI AUTO-RIFLESSIVITÀ}

Come abbiamo già accennato, negli ultimi venti anni la ricerca evidencebased ha prodotto considerevoli risultati sia relativamente alle strategie didattiche efficaci, sia relativamente all'efficacia dei metodi di formazione degli insegnanti.

John Hattie (2009 e 2012), noto per i suoi rilevanti apporti di sintesi sulla conoscenza delle didattiche efficaci, riconosce come maggiormente determinante dell'efficacia dell'azione didattica la qualità dell'interazione tra docente e allievo (in particolare il feedback e la valutazione formativa ${ }^{23}$ ) e sostiene che è necessario rendere "visibile» il processo di insegnamentoapprendimento. «La caratteristica notevole dell'evidenza è che i maggiori effetti sull'apprendimento degli studenti si verificano quando gli insegnanti diventano allievi del proprio insegnamento, e quando gli studenti diventano i propri insegnanti» (2009, p. 22, trad. libera). Il processo di insegnamento-apprendimento è visibile quando l'insegnante vede l'apprendimento attraverso gli occhi degli studenti e lo studente si vede con gli occhi del suo insegnante. Il modello di insegnamento-apprendimento visibile combina al suo interno l'insegnamento centrato sull'insegnante, ossia l'impostazione didattica, e l'apprendimento centrato sullo studente, ossia la partecipazione attiva dello studente, in una dialettica tra istruzione diretta e costruzione della conoscenza.

Le risultanze della ricerca educativa evidence based suggeriscono che i metodi più adeguati per la formazione degli insegnanti sono quelli che prevedono situazioni pratiche e concrete (laboratory experiences) accompagnate da attività di riflessione e confronto critico (coaching and feedback), anche con momenti di auto-osservazione (Hattie, 2009, p. 112). Metcalf (1995 - citato in Hattie, 2009) dalla meta-analisi compiuta sulle pratiche di formazione degli insegnanti, conclude che le esperienze pratiche producono una scala di effetti di rilievo $\left(E S=0,70^{24}\right)$ e il loro effetto non si riduce nel tempo. Bennett (1987 - citato in Hattie, 2009) sostiene che, affinché un metodo di formazione per gli insegnanti sia efficace, è necessario che includa teoria, dimostrazione e pratica, nonché feedback, preferibilmente distribuiti in varie sessioni.

23 Sono dati in linea con i profili di didattica efficace quali quelli già avanzati da Gagné, Merrill, Rosenshine, e dalla teoria del carico cognitivo (cfr. Calvani, 2011e 2012).

24 L'Effect Size è un valore, calcolato in unità di deviazione standard, che indica la differenza tra i risultati ottenuti dal gruppo sperimentale e quelli ottenuti dal gruppo di controllo (Hattie, 2009 e 2012; Calvani, 2012). Un Effect Size è ritenuto accettabile quando è maggiore di 0,4 (ibid.). 
Il video apre nuove prospettive per la formazione insegnanti: si rivela un espediente di successo in grado di integrare e supportare, attraverso la dimensione visiva, l'osservazione pratica e diretta, l'insegnamento-apprendimento di buone pratiche didattiche che altrimenti rimarrebbero spiegate solo verbalmente. Diversi studi attestano l'efficacia di interventi video-based nella formazione degli insegnanti (Santagata, Zannoni, \& Stigler, 2007). Il video è stato riconosciuto come (i) valido all'interno di pratiche di modellamento, in quanto non soltanto fornisce un'esemplificazione della pratica, ma impegna gli studenti in un processo di analisi complesso e riflessivo (Santagata \& Guarino, 2011); (ii) utile promotore di una riflessione generale e personale - nel caso di auto-analisi - sulla didattica (ad esempio nella pratica del microteaching) (van Es \& Sherin, 2002); (iii) efficace strumento in grado di far focalizzare l'attenzione sugli studenti (ad esempio nel video club) (Franke et al., 2001; Santagata et al., 2007; van Es \& Sherin, 2008).

In ciascuno dei casi presentati poco sopra, l'utilizzo del video per la formazione insegnanti risulta efficace se proposto all'interno di un'attività ben strutturata. Secondo il modello quadripartito presentato da Santagata (2012) è importante prima di tutto (i) definire chiaramente gli obiettivi di apprendimento che si intendono conseguire con il gruppo di insegnanti in formazione; (ii) selezionare la/e tipologia/e di video che rispondono meglio agli obiettivi posti (i criteri sono: focus, durata, montaggio, attori, tipo di insegnamento); (iii) suggerire agli insegnanti una guida per la visione del video (a questo scopo può essere utile il Lesson Framework Analysis ${ }^{25}$; Santagata \& Angelici, 2010); (iv) elaborare strumenti di valutazione allineati agli obiettivi, dai quali, in un processo ciclico, trarre informazioni per una nuova riformulazione del corso (Santagata, 2012).

Il microteaching (letteralmente "micro-insegnamento»), che ottiene un indice di efficacia molto alto $(E S=0,88)$, è una tecnica che è stata elaborata e messa in pratica per la prima volta nel 1963 all'Università di Stanford in un programma rivolto alla formazione degli insegnanti (Allen, 1967; Cooper \& Allen, 1970); è un insegnamento in situazione per un numero ridotto di tempo (da 5 a 25 minuti circa) e con pochi studenti (da 5 a 10 massimo) che solitamente viene videoregistrato (o audioregistrato) (Allen \& Clark, 1967).

25 Il Lesson Framework Analysis, realizzato per guidare l'osservazione dei video da parte degli insegnanti in formazione, si fonda su tre domande principali: (i) Qual è l'obiettivo di apprendimento principale della lezione (o del segmento visionato)?; (ii) Gli studenti hanno fatto progressi verso tale obiettivo? Come è possibile affermare ciò? Quali dati lo suggeriscono? Quali dati risultano mancanti?; (iii) Quali attività o decisioni dell'insegnante risultano efficaci e quali, invece, no? Come migliorare la lezione per renderla più efficace dal punto di vista dell'apprendimento degli studenti? 
Esso offre agli insegnanti la possibilità di lavorare in situazioni pratiche e reali, di rivedersi da "fuori» e di ottenere una serie di feedback (dai colleghi, da altri esperti e/o dal mentore) che gli consentono di migliorare le proprie capacità di insegnamento e potenzialmente di re-insegnare la stessa lezione in modo più efficace, anche a un gruppo più grande (Borg et al., 1969; Calvani, Bonaiuti, \& Andreocci, 2011; Bonaiuti, Calvani, \& Picci, 2012).

Un intervento di microteaching si articola in più fasi:

- progettazione della micro-lezione (plan);

- realizzazione e videoregistrazione della micro-lezione in classe (teach);

- osservazione della videoregistrazione da parte dello stesso insegnante e di un gruppo di colleghi/esperti e/o mentore (critique);

- riprogettazione della micro-lezione alla luce di quanto emerso dai feedback ricevuti (re-plan);

- realizzazione di un nuovo intervento didattico nella sua versione modificata in un'altra classe (re-teach);

- osservazione della nuova micro-lezione e nuova sessione di feedback da parte del medesimo gruppo di colleghi/esperti e/o mentore (critique) (Cooper \& Allen, 1970).

Affinché le osservazioni delle videoregistrazioni siano efficaci e non dispersive, in ogni sessione di feedback è necessario focalizzare l'attenzione su uno, al massimo due, specifici elementi sui quali poi il gruppo di colleghi, esperti e/o il mentore deciderà di lavorare (Allen, 1967).

La scelta di lavorare «in situazione», ossia in una classe vera, consente ai neofiti di impegnarsi nella pratica attivamente e di sperimentare fin da subito la relazione con gli studenti e i vari livelli di età (Allen \& Clark, 1967), quella di semplificare la complessità del regolare processo di insegnamento-apprendimento è orientata a fornire ambienti di formazione ottimale. Lidea fondamentale che sta alla base è che la situazione di insegnamento reale, seppur con difficoltà ridotta, stimoli un impegno e un coinvolgimento attivo, tenendo allo stesso tempo bassa la minaccia dell'ansia e il rischio sia per l'insegnante che per lo studente (ibid.; Cooper, 1967; Johnson, 1967; Cooper \& Allen, 1970). La brevità della lezione poi consente un'analisi intensa e attenta della videoregistrazione (Allen, 1967).

La scelta di videoregistrare l'intervento didattico, sebbene non sia un elemento essenziale del microteaching, offre notevoli e importanti opportunità a questa pratica. In particolare, risulta essere determinante il potere di ripresentare, anche a distanza di tempo, in maniera invariata e oggettiva, un evento, consentendo: all'insegnante in formazione di potersi rivedere da «fuori», con occhio esterno e/o dal punto di vista degli allievi, e ad altri (colleghi, mentore, esperti, ma anche ricercatori) di poter osservare direttamente (senza ulteriori mediazioni) l'operato dell'insegnante (Allen, 1967; Allen \& Clark, 1967). Secondo Cooper (1967) il fatto di rivedersi sul monitor di una 
televisione aiuta l'insegnante a prendere le distanze dal comportamento messo in atto e a percepire eventuali critiche mosse in modo meno difensivo.

Ciò che è il vero fulcro del microteaching è il ciclo teach-critique e re-teach-critique che può essere ripetuto anche più volte (ibid.; Cooper, 1967; Cooper \& Allen, 1970). I feedback, formulati da colleghi, mentore o esperti immediatamente dopo l'osservazione della videoregistrazione, orientano la riflessione professionale e l'acquisizione di consapevolezza circa le potenzialità e i punti deboli dimostrati e consentono all'insegnante di riformulare in maniera più adeguata una nuova micro-lezione (Allen \& Clark, 1967; Borg et al., 1969; Cooper \& Allen, 1970; Calvani et al., 2011; Bonaiuti et al., 2012). In questo modo viene offerta all'insegnante, soprattutto se in formazione, l'opportunità di fare esperienza di diversi contesti, varie tipologie di allievi e diversi livelli di età, per promuovere lo sviluppo di una più vasta gamma di competenze e abilità (Allen \& Clark, 1967).

È interessante vedere che per la formazione dei futuri insegnanti è prevista in alcuni casi anche una fase preliminare alle altre poco sopra descritte, in cui vengono fornite indicazioni sulle competenze da sviluppare. Queste indicazioni possono essere scritte, spiegate oralmente o dimostrate attraverso video di insegnanti esperti che fungono da modello (Cooper, 1967; Cooper \& Allen, 1970).

Sebbene inizialmente il microteaching fosse indirizzato quasi esclusivamente a insegnanti in formazione, si è configurato in seguito anche come metodo di formazione per insegnanti in servizio, metodo di sperimentazione di nuove tecniche o modalità di presentazione come strumento adatto ad esplorare gli effetti del processo di insegnamento-apprendimento in condizioni controllate (Allen, 1967; Allen \& Clark, 1967; Cooper \& Allen, 1970). Agli insegnanti in servizio il microteaching può offrire occasioni di esercizio all'osservazione e analisi dei comportamenti degli insegnanti principianti nonché un'occasione di confronto tra colleghi (Cooper \& Allen, 1970).

Oggigiorno, grazie all'avvento del digitale, il microteaching risulta potenziato da nuove funzionalità, che non potevano esistere quando vi era la videoregistrazione analogica. Tra queste vanno citate la possibilità di collaborare a distanza (Calvani et al., 2011; Bonaiuti et al., 2012; Picci et al., 2012; Santagata, 2012) e la video annotazione (Preston et al., 2005; Calvani et al., 2011; Bonaiuti et al., 2012; Picci et al., 2012). La video annotazione è una funzione presente in vari software che permette di associare un commento testuale ai singoli fotogrammi del video, offrendo così la possibilità di ancorare annotazioni a istanti specifici della videoregistrazione (Preston et al., 2005; Rich \& Hannafin, 2009) ${ }^{26}$.

26 Per una rassegna sugli strumenti di video annotazione cfr. Rich \& Hannafin, 2009. 


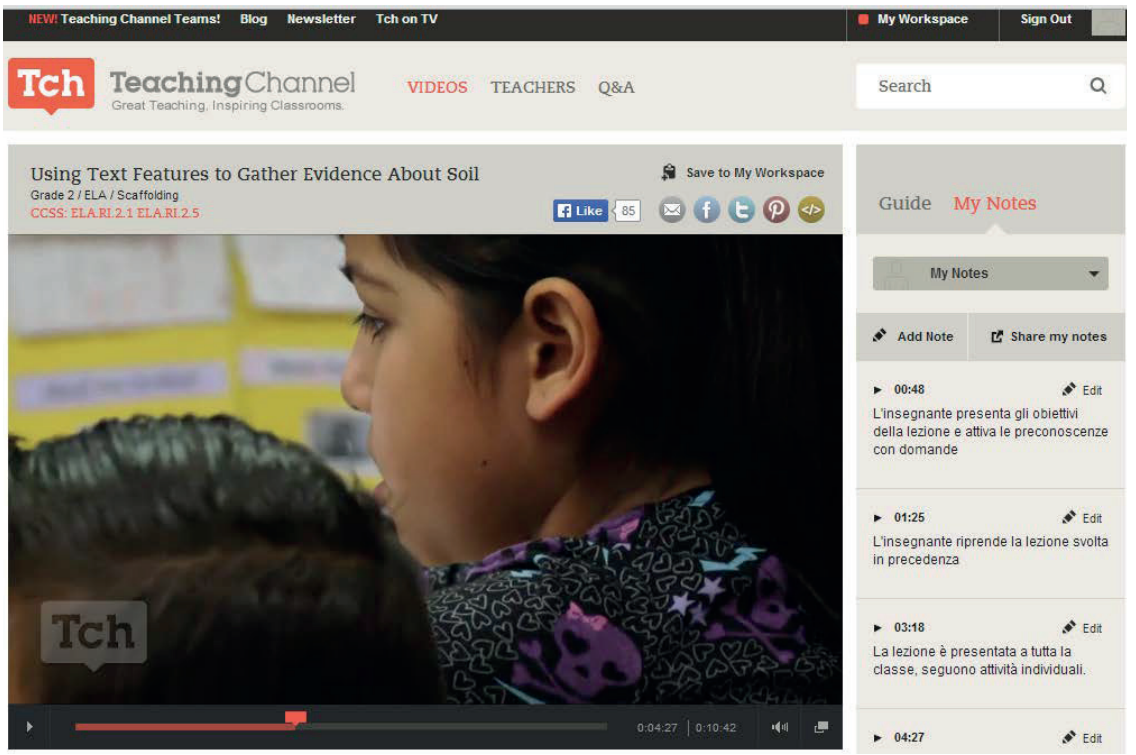

Figura 1. - Esempio di video annotazione sulla piattaforma di Teaching Channel.

Teaching Channel, tra le piattaforme di video-sharing presentate, offre ai suoi iscritti la possibilità di inserire annotazioni scritte legate a singoli frammenti video. Queste sono elencate con il riferimento al frammento video sulla destra, sotto la sezione "My Notes», e condivisibili o meno con la community del sito (Figura 1).

Recentemente sono nati e diffusi, soprattutto negli Stati Uniti, i video club (van Es \& Sherin, 2008). I video club sono gruppi di insegnanti, guidati da un preciso obiettivo e affiancati da un facilitatore ${ }^{27}$, che $s i$ incontrano regolarmente per osservare e discutere segmenti di video registrati all'interno delle loro classi (van Es, 2010). I video club sono particolarmente focalizzati sull'analisi del ragionamento e del pensiero degli studenti, che solitamente sfugge agli insegnanti durante le normali interazioni didattiche in classe (ibid.; Santagata, 2012). Dai primi studi effettuati sul campo, gli insegnanti partecipanti ai video club risultano aver acquisito maggiori capacità nel prestare attenzione al pensiero degli allievi, e non solo al comportamento e allo

${ }^{27}$ Il ruolo del facilitatore può essere assunto dal dirigente scolastico, un formatore ma anche da un membro del gruppo stesso. 
stato d'animo degli stessi, nello stimolare negli studenti un pensiero approfondito e nell'invitarli a esprimerlo verbalmente, rendendolo «visibile» (van Es, 2010). Queste azioni portano a una maggiore consapevolezza sulle idee dei propri allievi spingendo gli insegnanti partecipanti a ripensare l'insegnamento (ibid.).

È importante notare che uno dei principali vincoli dei video club, che sostanzialmente ne consente il successo, è il fatto che gli insegnanti coinvolti siano fermamente concentrati a riconoscere positivamente e a criticare produttivamente gli sforzi del lavoro del collega videoregistrato (ibid.). Il ruolo del facilitatore ha anche questo importante compito: fare in modo che questo vincolo venga rispettato e la discussione non proceda fuori dagli obiettivi e dal focus (ibid.).

\section{IL VIDEO-MODELING: ASPETTI CRITICI}

Come abbiamo visto oggigiorno il web offre un'impressionante ricchezza di risorse video. Questo esponenziale incremento è stato favorito essenzialmente dallo sviluppo tecnologico, nello specifico dalla diffusione della banda larga e dalla miniaturizzazione delle tecnologie, sempre più leggere, portatili e intuitive.

L'interesse significativo per questo ambito, dimostrato dal numero crescente di piattaforme di condivisione specifiche dedicate alla formazione (si veda par. su YouTube e altre piattaforme di video-sharing), costringe a fare il punto sulle tendenze nel processo di insegnamento-apprendimento mediato dai video e a interrogarsi circa alcune questioni ancora oggi in parte irrisolte.

Innanzitutto è necessario ricordare che, oltre alle piattaforme di videosharing presentate se ne possono trovare altre, e che non tutte possono essere ad accesso libero e gratuito. È importante poi conoscere le principali caratteristiche dei vari repository per poterli interrogare adeguatamente.

In una ricerca di un video sul web ${ }^{28}$ è determinante la selezione delle parole chiave. In questa fase è importante tenere conto che la maggior parte delle risorse video presenti sul web sono in lingua inglese. In lingua italiana, $o$ in altra lingua, è difficile trovare la ricchezza di contenuti che è possibile

${ }_{28}$ Qui si fa esclusivo riferimento a YouTube, piattaforma generalista, perché gli altri repository citati, avendo un numero di video relativamente ridotto, hanno filtri di ricerca diversi e specifici per il settore educativo (con possibilità di selezionare il grado scolastico di riferimento, l'area disciplinare, eventualmente la lingua o la tematica cui afferisce). 
invece reperire in lingua inglese (eccetto gli specifici repository nazionali). Per formulare una corretta query è altresì importante ricordare che una sola parola (ad es. shapes ${ }^{29}$ ) tende a generare un altissimo numero di record ${ }^{30}$, molti dei quali poco pertinenti alla nostra specifica richiesta. Per questa ragione, conviene sempre abbinare insieme più parole ${ }^{31}$ (ad es. 2-dimensional shapes preschool ${ }^{32}$ ), ottenendo una relativa riduzione dei record $^{33}$.

Tra i numerosi record che può restituire YouTube può essere utile per una prima selezione affidarsi ai filtri che mette a disposizione, ossia per data di caricamento, tipo di risultato (se video, canale playlist, filmato o program$\mathrm{ma}$ ), per durata (se non superiore ai 4 minuti o non superiore ai 20 minuti), per caratteristiche (alta definizione, sottotitoli, Creative Commons, 3D, dal vivo o acquistato), e con la possibilità di ordinarli per pertinenza, data di caricamento, numero di visualizzazioni o voto.

La fase di selezione dei risultati è altrettanto critica. Solitamente ci si limita a sfogliare le prime pagine ( 1 o al massimo 2 ) di record, ma non sempre queste rispondono adeguatamente alla nostra ricerca. Il rischio poi di dispersione è altissimo: per ogni video visionato ne vengono suggeriti altri (nella schermata finale del video appena visionato e sul lato destro della pagina), innescando una catena nella quale diventa difficile orientarsi. Per questa ragione risulta essere utile affidarsi ai filtri disponibili su YouTube e alle informazioni solitamente inserite per ogni singolo video. Il tempo di durata di un video è un elemento determinante se si ritiene di utilizzarlo didatticamente (un video troppo lungo rischia di essere dispersivo rispetto al contenuto dello stesso); la qualità del video influisce sull'appeal del video stesso (un video in bassa qualità, il cui audio è disturbato, risulta essere un ostacolo e non un espediente favorevole all'apprendimento). La qualità della fonte poi è un aspetto rilevante che determina molto spesso la buona riuscita della ricerca.

La facilità di accesso, caricamento e condivisione dei video sul web generano uno sviluppo esponenziale delle risorse disponibili, non mantenendo

29 In questo contesto possiamo tradurre in italiano la parola inglese shapes con l'espressione «forme geometriche».

30 Inserendo questa parola in YouTube si ottengono 2.520.000 risultati (consulted 26/08/2014).

31 Inserendo più parole nella ricerca, YouTube lega automaticamente quelle parole con l'operatore booleano AND, ossia consente di trovare tutte le risorse in cui compaiano contemporaneamente le parole indicate.

32 Possiamo tradurre in italiano questa espressione con «forme geometriche bidimensionali scuola dell'infanzia».

33 Inserendo queste parole chiave in YouTube si ottengono 2.900 risultati (consulted 26/08/2014). 
però alti standard di qualità. Buona parte dei video, infatti, è realizzata dai singoli utenti che popolano il web. Per questo motivo è importante interrogarsi sempre sulla provenienza della fonte e sulla sua qualità. Utile a questo scopo è verificare la natura del canale di provenienza ${ }^{34}$.

Un ulteriore problema dei video reperiti sul web è dettato dal fatto che molto spesso questo genere di video è realizzato in contesti di apprendimento lontani e differenti da quelli vissuti dall'insegnante in formazione (Gaudin \& Flandin, 2011). Questo potrebbe generare una sorta di «rifiuto» del video stesso e del messaggio didattico che intende trasmettere.

\section{IL VIDEO-COACHING: ASPETTI CRITICI}

Numerosi studi svolti nell'ambito del microteaching rivelano risultati significativi di questa pratica non solo in termini di efficacia, ma anche di efficienza, economizzando contemporaneamente tempo e risorse, favorendo l'apprendimento di un numero maggiore di competenze didattiche, l'approfondimento nella pratica di quelle abilità, avendo più tempo da dedicare a corsi, e un ruolo più efficace dei soggetti addetti alla supervisione (Allen $\&$ Clark, 1967). Nonostante questo il microteaching non si è affermato in maniera decisiva sulle altre pratiche didattiche. I principali ostacoli incontrati negli anni Settanta, Ottanta e Novanta riguardano le tecnologie: telecamere e supporti per la conservazione video (VHS) sono troppo costosi.

Dagli anni Duemila, grazie all'avvento delle tecnologie digitali, questi problemi possono essere superati. La digitalizzazione dei file consente la miniaturizzazione dei dispositivi, sia telecamere/videocamere che supporti di memoria (CD, DVD, driver USB, schede di memoria), rendendo, tra l'altro, ancora più facile la possibilità di editare, scambiare e condividere video in modo facile, veloce e gratuito. La forte diminuzione dei prezzi poi permette un'ampia diffusione dei dispositivi: basti pensare che, in questi ultimi anni, un numero crescente di persone ha acquistato un telefono cellulare, personal computer o tablet con una videocamera integrata.

Nonostante ciò continuano a conservarsi vari ostacoli ad una sua piena adozione, in particolare nel contesto italiano la difficoltà principale è da

34 Non tratteremo in questa sede dei problemi legati alla catalogazione e conservazione (quali supporti per la conservazione, quali metodi, nonché problemi concernenti i diritti d'autore), sebbene questa esigenza sia forte soprattutto per tutte quelle informazioni veicolate nel web, la cui volatilità (mutazioni di URL, chiusura di siti, ...) costringe a pensare e organizzare necessariamente un archivio, un repository di video nello specifico. 
ricercarsi nel disagio e nella difficoltà degli insegnanti a esporsi a proiezioni fuori dalle mura scolastiche. Da una ricerca di Bonaiuti et al. (2011) emerge che gli insegnanti, tra la possibilità di confrontarsi con il gruppo dei pari, un collega mentore di fiducia o un esperto da remoto, preferiscono ricevere feedback da un collega mentore di fiducia. La possibilità di confrontarsi con il gruppo dei pari, invece, risulta essere «la meno desiderabile» (ivi, p. 255). Questo probabilmente è legato al fatto che il rapporto di coppia rispetto al confronto con il gruppo dei pari è percepito come più "protetto", meno esposto alla condivisione pubblica che rischia di minacciare l'autostima personale. Il problema principale di questa tipologia di video è strettamente correlato alla paura del giudizio dell'altro (Gaudin \& Simon, 2011).

\section{ConClusioni}

La videoeducazione assume oggi con il Web 2.0 nuova dimensione e formato. I recenti sviluppi tecnologici (diffusione della banda larga, miniaturizzazione dei supporti di memoria e dei dispositivi tecnologici), nonché la semplicità d'uso e l'abbassamento dei costi delle tecnologie hanno contribuito all'esplosione del fenomeno dei video digitali, che oggi registra numeri in costante aumento.

Nell'ambito di questa considerevole crescita sia di fruizione che di produzione dei video digitali, nuovo interesse e nuove prospettive si delineano in relazione alla formazione degli insegnanti. I video consentono di potenziare i percorsi di formazione, tradizionalmente basati su trasmissione verbale, con momenti di osservazioni, di dimostrazioni pratiche e auto-analisi e riflessività.

In questo contributo ci siamo soffermati su due tipologie di video educativo impiegato con insegnanti: il video-modeling, generalmente prodotto da altri (insegnanti sconosciuti o colleghi) e il video-coaching, prodotto dall'insegnante stesso come termine di riflessione per migliorare il proprio comportamento didattico. Queste tipologie di video offrono differentemente vantaggi agli insegnanti in formazione, ma presentano al contempo dei limiti e delle problematiche.

I video-modeling esterni consentono di acquisire un metodo pratico di osservazione e analisi professionale, nonché di imparare buone pratiche, vedendo operativamente come queste vengono messe in atto. Questo genere di video però ha un limite: spesso i contesti di apprendimento sono lontani e differenti da quelli vissuti dall'insegnante in formazione (Simon \& Flandin, 2011). I video-modeling prodotti dai colleghi, invece, rassicurano gli insegnanti, rompendo l'isolamento in cui solitamente si trovano a lavorare e 
consentono di vedere ciò che fanno i colleghi, in realtà vicine e conosciute, impegnandoli in riflessioni positivamente critiche e comparative. Questa tipologia di video però può generare negli insegnanti una chiusura per la paura del giudizio dell'altro.

Ulteriori criticità sorgono nel caso in cui si intenda procedere alla ricerca sul web di video ad uso educativo. L'esplosione dei video digitali infatti, sebbene prospetti grandi potenzialità educative, al contempo produce dispersività e rischio di sovraccarico. In una ricerca su YouTube ci si può facilmente imbattere in un numero di risultati talmente consistente che necessita, affinché possa diventare gestibile, di essere ridotto grazie all'utilizzo degli appositi filtri, che qualche sito mette a disposizione di default, e grazie a buone capacità selettive dell'insegnante ricercatore. Il modello quadripartito presentato (Santagata, 2012) risulta utile ad orientare in questa fase. In ogni caso, affinché si possa beneficiare dell'enorme quantità di risorse disponibili online, sarà necessario sviluppare una digital literacy e una consapevolezza maggiore dei formatori universitari per poter organizzare anticipatamente la formazione degli insegnanti in questo settore.

I video autoprodotti hanno il principale vantaggio di stimolare l'autoosservazione e l'auto-analisi, favorendo la maturazione di nuove consapevolezze sul proprio modo di fare didattica e su ciò che solitamente sfugge facendo lezione (ad esempio i pensieri e le idee degli allievi) al fine di migliorarsi in un futuro prossimo. Molti insegnanti però vivono con disagio il rivedersi, soprattutto se questo avviene anche in presenza di altri (colleghi in particolare). Vi sono ancora resistenze tra gli insegnanti nell'aprirsi a scambi professionali, positivi e produttivi di criticità. Per questo motivo risulta opportuno promuovere fin dai primi anni del percorso formativo specifico per insegnanti incontri di gruppo e confronti critici a favore di una cultura ispirata alla piena trasparenza di ciò che l'insegnante fa nella propria classe.

\section{RIFERIMENTI BIBLIOGRAFICI}

Accenture (2012). Video-over-internet: Consumer survey 2012. Retrieved from: http:// www.accenture.com/it-it/Pages/insight-video-over-internet-consumer-survey-2012.aspx (consulted 03/2013).

Allen, D. W. (1967). Microteaching: A description. Stanford, CA: Stanford University. Retrieved from: http://files.eric.ed.gov/fulltext/ED019224.pdf (consulted 02/2013).

Allen, D. W., \& Clark, R. J. (1967). Microteaching: Its rationale. The High School Journal, 51(2), 75-79. Retrieved from: http://www.jstor.org/stable/40366699 (consulted 03/2013). 
Audiweb (2014a). Audiweb pubblica $i$ dati di audience online da PC del mese di febbraio 2014. Retrieved from: http://www.audiweb.it/cms/view.php?id=6\&cms $\mathrm{pk}=318$ (consulted 05/2014).

Audiweb (2014b). Audiweb pubblica $i$ dati di audience online da PC del mese di aprile 2014. Retrieved from: http://www.audiweb.it $/ \mathrm{cms} / \mathrm{view} \cdot \mathrm{php}$ ?id=4\&cms_ $\mathrm{pk}=320$ (consulted 05/2014).

Berelowitz, M., \& Palley, W. (2014). 10 mobile trends for 2014 and beyond. New York: JWT. Retrieved from:: http://www.jwt.com/en/worldwide/thinking/10 mobiletrendsfor2014andbeyond/ (consulted 06/2014).

Bonaiuti, G. (2010). Didattica attiva con i video digitali: metodi, tecnologie, strumenti per apprendere in classe e in Rete. Trento: Erickson.

Bonaiuti, G., Calvani, A., \& Picci, P. (2012). Tutorship e video annotazione: il punto di vista degli insegnanti / Tutorship and video annotation: teachers' perspectives on innovation. Giornale Italiano della Ricerca Educativa, 9736, 246-258.

Borg, W. R., Kallenbach, W., Morris, M., \& Friebel, A. (1969). Videotape feedback and microteaching in a teacher training model. The Journal of Experimental Education, 37(4), 9-16.

Calvani, A. (2007). Evidence-based education: ma "funziona» il "che cosa funziona»? Je-LKS. Journal of e-Learning and Knowledge Society, 3(3), 139-146. Retrieved from: http://www.je-lks.org/ojs/index.php?journal=Je-LKS_

$\mathrm{EN} \&$ page $=$ article\&op $=$ view \& path $\% 5 \mathrm{~B} \% 5 \mathrm{D}=766 \&$ path $\% 5 \mathrm{~B} \% 5 \mathrm{D}=765$ (consulted 04/2014).

Calvani, A. (a cura di). (2011). Principi dell'istruzione e strategie per insegnare: Criteri per una didattica efficace. Roma: Carocci.

Calvani, A. (2012). Per un'istruzione evidence based. Analisi teorico-metodologica internazionale sulle didattiche efficaci e inclusive. Trento: Erickson.

Calvani, A., Bonaiuti, G., \& Andreocci, B. (2011). Il microteaching rinascerà a nuova vita? Video annotazione e sviluppo della riflessività del docente. Giornale Italiano della Ricerca Educativa, 4(6), 29-42.

Cooper, J. M. (1967). Developing specific teaching skills through micro-teaching. The High School Journal, 51(2), 80-85. Retrieved from: http://www.jstor.org/ stable/40366700 (consulted 04/2014).

Cooper, J. M., \& Allen, D. W. (1970). Microteaching: history and present status. Washington: ERIC Clearing House on Teacher Education. Retrieved from: http://files.eric.ed.gov/fulltext/ED036471.pdf (consulted 04/2014).

Dordit, L. (2011). Modelli di reclutamento, formazione, sviluppo e valutazione degli insegnanti. Breve rassegna internazionale. Trento: IPRASE Trentino.

European Commission (2002). Questioni chiave dell'istruzione in Europa. La professione docente in Europa: profili, tendenze e sfide. Rapporto I: Formazione iniziale e passaggio alla vita professionale. Bruxelles: Eurydice. Retrieved from: file://C:/ Users/pc/Downloads/EC3012565ITN_002.pdf (consulted 08/2014). 
European Commission/EACEA/Eurydice (2013). Key data on teachers and school leaders in Europe. 2013 Edition. Eurydice Report. Luxembourg: Publications Office of the European Union. Retrieved from: http://eacea.ec.europa.eu/education/ eurydice/documents/key_data_series/151EN.pdf (consulted 08/2014).

Franke, M. L., Carpenter, T. P., Levi, L., \& Fennema, E. (2001). Capturing teachers' generative change: A follow-up study of professional development in mathematics. American Educational Research Journal, 38(3), 653-689. Retrieved from: http://aer.sagepub.com/content/38/3/653.full.pdf+html (consulted 08/2014).

Gaudin, C., \& Flandin, S. (2011). La vidéoformation dans tous ses états: Quelles options théoriques? Quels scénarios? Pour quels effets? Conférence de consensus, 23 Janvier 2014, Chair UNESCO: Former les einsegnants au XXI siècle. Retrieved from: http://www.ens-lyon.fr/chaire-unesco-formation/manifestations-scientifiques/video-formation/conference-sur-la-videoformation/etatde-l-art-s-flandin-et-c-gaudin (consulted 08/2014).

Gutiérrez Martín, A. (Ed.). (2011). Video education, media education and lifelong learning: A European insight. Kulturring E. V. Berlino. Retrieved from: http:// viducate.net/attachments/101_viducatePublication2011OnlineVersion.pdf (consulted 04/2013).

Hargreaves, D. H. (1997). In defence of research for evidence-based teaching: A rejoinder to Martyn Hammersley. British Educational Research Journal, 23, 405-419.

Hattie, J. (2009). Visible Learning: A synthesis of over 800 meta-analyses relating to achievement. London - New York: Routledge.

Hattie, J. (2012). Visible learning for teachers: Maximizing impact on learning. London - New York: Routledge.

ILO - International Labour Organization (2010). Teachers and trainers for the future. Technical and vocational education and training in a changing world. Genève. Retrieved from: http://www.ilo.org/public/libdoc/ilo/2010/460299. pdf (consulted 08/2014).

Jarboe, G. (2014). comScore: Video ad views hit 26.9 billion in January. http://www. clickz.com/clickz/news/2330651/comscore-video-ad-views-hit-269-billionin-january (consulted 05/2014).

Johnson, W. D. (1967). Microteaching: A medium in which to study teaching. The High School Journal, 51(2), 86-92. Retrieved from: http://www.jstor.org/ stable/40366701 (consulted 04/2014).

Kaufman, P. B., \& Mohan, J. (2009). Video use and higher education: Options for the future. Retrieved from: http://library.nyu.edu/about/Video_Use_in_Higher_ Education.pdf (consulted 03/2013).

Masats, D., \& Dooly, M. (2011). Rethinking the use of video in teacher education: A holistic approach. Teaching and Teacher Education, 27, 1151-1162. Retrieved from: http://ictandenglish.webs.com/documents/Rethinking\%20 
the\%20use \%20of\%20video\%20in\%20teacher\%20education.pdf (consulted 08/2014).

Mayer, R. E. (2001). Multimedia learning. Cambridge: Cambridge University Press.

Metcalf, K. K. (1995). Laboratory experiences in teacher education: A meta-analytic review of research. Paper presented at the Annual Meeting of the American Educational Research Association, San Francisco. Retrieved from: http:// www.eric.ed.gov/PDFS/ED388645.pdf (consulted 03/2013).

Negroponte, N. (1995). Being digital. Trad. it. a cura di F. e G. Filippazzi (ed. orig. 1995). Milano: Sperling \& Kupfer.

OECD - Organization for Economic and Co-operation Development (2005). Attracting, developing and retaining effective teachers. Final report: Teachers Matter. Retrieved from: http://www.oecd.org/education/school/ attractingdevelopingandretainingeffectiveteachers-finalreportteachersmatter. htm (consulted 08/2014).

OECD - Organization for Economic and Co-operation Development (2009). Evaluating and rewarding the quality of teachers: International practices. Retrieved from: http://www.oecd.org/fr/edu/scolaire/

evaluatingandrewardingthequalityofteachersinternationalpractices.htm (consulted 08/2014).

O'Reilly, T. (2005). What is web 2.0? Design patterns and business models for the next generation of software. Retrieved from: http://oreilly.com/web2/archive/whatis-web-20.html (consulted 03/2014).

Picci, P., Calvani, A., \& Bonaiuti, G. (2012). The use of digital video annotation in teacher training: The teachers' perspectives. Procedia - Social and Behavioral Sciences, Special Issue: International Conference on Education and Educational Psychology (IEEPSY 2012), 69.

Preston, M. D., Campbell, G. A., Ginsburg, H. P., Sommer, P., \& Moretti, F. A. (2005). Developing new tools for video analysis and communication to promote critical thinking. Paper presented at the ED-MEDIA World Conference on educational multimedia, hypermedia and telecommunications, Montreal, 2 July. Retrieved from: http://www.editlib.org/noaccess/20763/ (consulted 03/2014).

Ranieri, M. (2007). Evidence Based Education: un dibattito in corso. Je-LKS. Journal of e-Learning and Knowledge Society, 3(3), 147-152. Retrieved from: http:// www.je-lks.org/ojs/index.php?journal=Je-LKS_EN\&page=article\&op=view \&path\%5B\%5D=767\&path\%5B\%5D=766 (consulted 04/2014).

Rich, P. J., \& Hannafin, M. J. (2009). Video annotation tools: Technologies to scaffold, structure, and transform teacher reflection. Journal of Teacher Education, 60(1), 52-67. Retrieved from: http://jte.sagepub.com/content/60/1/52.full. pdf + html (consulted 04/2014). 
Santagata, R., \& Angelici, G. (2010). Studying the impact of the lesson analysis framework on preservice teachers' abilities to reflect on videos of classroom teaching. Journal of Teacher Education, 61(4), 339-349.

Santagata, R. (2012). Un modello per l'utilizzo del video nella formazione professionale degli insegnanti.Form@re - Open Journal per la Formazione in Rete, 79(12), 58-63. doi: http://dx.doi.org/10.13128/formare-12601 (consulted 04/2014).

Santagata, R., \& Guarino, J. (2011). Using the video to teach future teachers to learn from teaching. ZDM. The International Journal of Mathematics Education, 43(1), 133-145. doi: http://dx.doi.org/10.1007/s11858-010-0292-3.

Santagata, R., Zannoni, C., \& Stigler, J. W. (2007). The role of lesson analysis in pre-service teacher education: An empirical investigation of teacher learning from a virtual-based field experience. Journal of Mathematics Teacher Education, 10(2), 123-140. doi: http://dx.doi.org/10.1007/s10857-007-9029-9.

Sweller, J. (1988). Cognitive load during problem solving: Effects on learning. Cognitive Science, 12, 257-285. Retrieved from: http://csjarchive.cogsci.rpi. edu/1988v12/i02/p0257p0285/main.pdf (consulted 03/2014).

UNESCO (2005). Guidelines and recommendations for reorienting teacher education to address sustainability. Technical paper, 2. Paris. Retrieved from: http://unesdoc.unesco.org/images/0014/001433.20143370e.pdf (consulted 08/2014).

van Es, E. (2010). Viewer discussion is advised. Video clubs focus teacher discussion on student learning. JSD, 31(1).

van Es, E., \& Sherin, M. G. (2002). Learning to notice: Scaffolding new teachers' interpretations of classroom interactions. Journal of Technology and Teacher Education, 10(4), 571-596.

van Es, E., \& Sherin, M. G. (2008). Mathematics teachers" «learning to notice» in the context of a video club. Teaching and Teacher Education, 24, 244-276.

Zickuhr, K., \& Smith, A. (2012). Digital differences: While increased internet adoption and the rise of mobile connectivity have reduced many gaps in technology access over the past decade, for some groups digital disparities still remain. Retrieved from: http://www.pewinternet.org/ //media//Files/Reports/2012/ PIP_Digital_differences_041312.pdf (consulted 04/2014).

\section{SitOGRAFIA}

BBC News (canale YouTube), https://www.youtube.com/user/bbcnews (consulted 05/2014).

CERN (canale YouTube), https://www.youtube.com/user/CERNTV (consulted 05/2014). 
Coursera, https://www.coursera.org/ (consulted 05/2014).

Education Endowment Foundation, http://educationendowmentfoundation.org.uk/ toolkit/videos-case-studies/ (consulted 05/2014).

Edutopia (canale YouTube), https://www.youtube.com/user/edutopia (consulted 05/2014).

Edutopia, http://www.edutopia.org/videos (consulted 05/2014).

EduTubePlus, http://www.edutubeplus.info/project/edutubeplus (consulted 05/2014).

EdX, https://www.edx.org/ (consulted 05/2014).

EuTube (canale YouTube), https://www.youtube.com/user/eutube (08/2014).

Facebook, https://www.facebook.com/ (consulted 05/2014).

FrontBack, http://frontback.me (consulted 05/2014).

Google, http://www.google.com/ (consulted 08/2014).

Instagram, http://instagram.com/ (consulted 05/2014).

KeepVid, http://keepvid.com/ (consulted 08/2014).

Khan Academy (canale YouTube), https://www.youtube.com/user/khanacademy (consulted 08/2014).

La7 (canale YouTube), https://www.youtube.com/user/la7 (consulted 05/2014).

MIT (canale YouTube), https://www.youtube.com/user/MIT (consulted 05/2014).

MIUR (canale YouTube), https://www.youtube.com/user/ministeroistruzione (consulted 05/2014).

Numberphile (canale YouTube), https://www.youtube.com/user/numberphile (consulted 08/2014).

OnAir, http://www.onair.medmediaeducation.it/ (consulted 05/2014).

PBS Kids, http://pbskids.org/ (consulted 08/2014).

PBS Learning Media (canale YouTube), https://www.youtube.com/user/pbslearningmedia (consulted 08/2014).

PBS Learning Media, http://www.pbslearningmedia.org/ (consulted 08/2014).

PBS Parents, http://www.pbs.org/parents/ (consulted 08/2014).

PBS Video, http://www.video.pbs.org (consulted 08/2014).

PBS, http://www.pbs.org/ (consulted 05/2014).

Pinterest, https://it.pinterest.com/ (consulted 05/2014).

Rai (canale YouTube), https://www.youtube.com/user/rai (consulted 05/2014).

SaveMedia, http://savemedia.com/ (consulted 08/2014).

Snapchat, https://www.snapchat.com/ (consulted 05/2014).

Standford University (canale YouTube), https://www.youtube.com/user/StanfordUniversity (consulted 08/2014).

Steve Spangler Science (canale YouTube), https://www.youtube.com/user/TheSpanglerEffect (consulted 08/2014). 
Teaching Channel, https://www.teachingchannel.org/ (consulted 05/2014).

TED Talk (canale YouTube), https://www.youtube.com/user/TEDtalksDirector (consulted 08/2014).

TED, http://www.ted.com/ (consulted 05/2014).

TED-Ed, http://ed.ted.com/ (consulted 08/2014).

TIMSS Video, http://www.timssvideo.com/ (consulted 05/2014).

Università di Firenze (canale YouTube), https://www.youtube.com/user/UNIFIRENZE (consulted 05/2014).

Viducate, http://viducate.net/ (consulted 05/2014).

Vimeo, https://vimeo.com/ (consulted 05/2014).

Vine, https://vine.co/ (consulted 05/2014).

YouTube, https://www.youtube.com/ (consulted 05/2014).

YouTube EDU, http://www.youtube.com/education (consulted 05/2014).

YouTube for School, http://www.youtube.com/schools (consulted 05/2014).

YouTube Statistics, https://www.youtube.com/yt/press/it/statistics.html (consulted 05/2014).

YouTube Teachers, http://www.youtube.com/user/teachers (consulted 05/2014).

\section{RiassunTO}

In un'accezione lata, la videoeducazione ha una lunga storia che risale allinizio del secolo scorso. La diffusione della televisione e, qualche anno più tardi, della multimedialità hanno segnato profondamente l'ascesa del video a livello educativo e didattico. In questi ultimi anni la digitalizzazione e la banda larga, nonché la sempre più diffusa disponibilità di tecnologie portatili, economiche e facili da utilizzare, hanno trasformato il web in un enorme luogo di produzione e condivisione di video. Il fenomeno oggi assume un nuovo risvolto anche per l'educazione ed appare destinato a modificare sensibilmente i metodi della formazione degli insegnanti. In questo contributo si descrivono le nuove prospettive della videoeducazione aperte dai recenti sviluppi del web, con particolare riferimento al Web 2.0 con YouTube e le altre piattaforme di video sharing. Verranno presentati due metodi di formazione supportati dall'uso del video: il "video-modeling» e il «video-coaching», analizzandone potenzialità e criticità, in riferimento alla formazione degli insegnanti.

Parole chiave: Formazione insegnanti, Video-coaching, Video digitali, Videomodeling, Web 2.0. 